\title{
Detection Probability Analysis of True Random Coding Photon Counting Lidar
}

\author{
Yang Yu ${ }^{1}$, Zhangjun Wang ${ }^{1, *}$, Kuntai Ma ${ }^{2}$, Chao Chen ${ }^{1}$, Xiufen Wang ${ }^{1}$, Boyang Xue ${ }^{1}$, Xianxin Li ${ }^{1}$, Feng Zhang ${ }^{1}$, \\ Xin Pan ${ }^{1}$, Quanfeng Zhuang ${ }^{1}$ and Hui $\mathrm{Li}^{1}$ \\ 1 Institute of Oceanographic Instrumentation, Qilu University of Technology (Shandong Academy of Sciences), \\ Qingdao 266001, China; yuyang@qlu.edu.cn (Y.Y.); chenchao@qlu.edu.cn (C.C.); \\ wxfsun2005@qlu.edu.cn (X.W.); byxue@qlu.edu.cn (B.X.); xianxinli@qlu.edu.cn (X.L.); \\ ffengzhang@126.com (F.Z.); xinnpan@yeah.net (X.P.); zhuangqf@qlu.edu.cn (Q.Z.); lihui@qlu.edu.cn (H.L.) \\ 2 College of Information Science \& Engineering, Ocean University of China, Qingdao 266100, China; \\ kuntaima@163.com \\ * Correspondence: zhangjunwang@qlu.edu.cn
}

Citation: Yu, Y.; Wang, Z.; Ma, K.; Chen, C.; Wang, X.; Xue, B.; Li, X.; Zhang, F.; Pan, X.; Zhuang, Q.; et al. Detection Probability Analysis of True Random Coding Photon Counting Lidar. Photonics 2021, 8, 545. https://doi.org/10.3390/ photonics 8120545

Received: 2 October 2021

Accepted: 26 November 2021

Published: 30 November 2021

Publisher's Note: MDPI stays neutral with regard to jurisdictional claims in published maps and institutional affiliations.

Copyright: (C) 2021 by the authors Licensee MDPI, Basel, Switzerland. This article is an open access article distributed under the terms and conditions of the Creative Commons Attribution (CC BY) license (https:// creativecommons.org/licenses/by/ $4.0 /)$.

\begin{abstract}
With the wide application of lidar in the future, the problem of crosstalk between lidars will become more serious. True random coding photon counting lidar with high anti-crosstalk ability will play an important role in solving this problem. In this paper, based on the working principle of Gm-APD, the detection probability theoretical model of true random coding photon counting lidar is built, and the impact of jitter on detection probability is considered for the first time. The influence of mean echo photon number, mean pulse count density, sequence length and pulse width on detection probability is analyzed. Monte Carlo simulation and experimental results are highly consistent with the theoretical model, which proves the correctness of the detection probability theoretical model. This theoretical model provides an effective means to evaluate the system performance.
\end{abstract}

Keywords: photon-counting; efficient imaging; noise robust; first signal photon unit; LIDAR

\section{Introduction}

Lidar has gradually become a promising means of environmental perception compared with traditional microwave radar due to its superior spatial and temporal resolution and the advantages of being less restricted by weather and lighting conditions. Especially photon counting lidar, due to its extremely high detection sensitivity, has attracted wide attention from researchers and has broad application prospects in the fields of ranging, three-dimensional imaging, target tracking and recognition, and mapping [1-5]. However, because the traditional photon counting lidar emits pulses with a fixed repetition frequency [6], the periodicity and regularity of the pulses will not only cause range ambiguity but are also vulnerable to interference and jamming. Especially in the near future, with the intensive application of lidar, it will be inevitable to receive signals from other lidars. In this case, the lidar will be affected by crosstalk, which will lead to the failure of detection $[7,8]$. Therefore, lidar should not only have high ranging accuracy and long ranging capability but also have strong anti-interference ability to meet the application requirements of complex scenarios in the future.

To mitigate the possibility of interference, modulation coded lidars have been studied. Lidar systems with different aperiodic or irregular laser pulse modulation schemes, such as the pseudo-random modulation [8-10] and the chaotic pulse position modulation (CPPM) have been studied [11-15]. However, the pseudo-random modulation sequence is periodic and reproducible, so a malicious jammer can easily record the transmitting pseudo-random modulation sequence and then re-transmit it to generate false echo to interfere with the lidar [12]. The CPPM sequence generated by chaotic map is susceptive to the change in parameters [15], and the chaotic pulse sequence generated based on the optical feedback method is complex and low in efficiency. 
In 2020, our previous work and Tsai almost simultaneously published a study on a new modulation method. In this method, Gm-APDs (Geiger-mode of Avalanche Photodiode) is used as random signal generators to generate true nature-based random sequences [16-18]. This true random modulation method has higher anti-interference immunity than the pseudorandom modulation method because the true random sequence generated based on Gm-APD is essentially a kind of digital noise, which is not repeatable and non- reproducible.

The peculiarity of this paper is using Gm-APD to generate true random sequence, which has strong anti-interference ability. Compared with Pseudorandom Noise (PN) sequence, it also has the advantage of overcoming the influence of Gm-APD dead time. For PN sequences, the interval between two adjacent ' 1 ' codes is less than the Gm-APD's dead time. When the Gm-APD's responds to a photon, it will enter the dead zone and cannot respond to any other arriving photons. Thus, when a ' 1 ' code of the PN sequence is responded, Gm-APD enters the dead zone, and any ' 1 ' code in the subsequent pseudorandom sequence cannot be responded until Gm-APD recovers from the dead zone [19-21]. Therefore, the ranging performance is degraded. For our method, a Gm-APD will not generate any pulse during its dead time, which means that the output pulse interval between any two adjacent pulses is larger than its dead time. Consequently, we can consider that any two ' 1 ' codes in the true random sequence are independent of each other $[17,18]$. In this case, the dead time effect can be completely avoided. The detection performance of true random coding photon counting lidar can be obviously improved.

Compared with the chaotic pulse position modulation method, the true random modulation method only needs one Gm-APD to generate a stable true random sequence. Moreover, the generated true random sequence can be directly used to drive the laser source to generate true random optical pulse sequence. The system is simpler than that of the chaotic pulse position modulation method.

In short, the true random coding photon counting lidar based on Gm-APD has the advantages of being a simple system and having a strong anti-crosstalk ability.

Tsai and Hwang verified that Gm-APD can not only produce high-quality random sequences, but also, the generated sequences have strong interference suppression capabilities [16,22,23]. Yu established the theoretical model of transmitting and receiving signals of the photon counting lidar system based on Gm-APD random coding, gave the function model of using system parameters to characterize the true random sequence autocorrelation, and preliminarily verified the detection performance of the system [18]. In addition, Liu's experiments verify the ranging and imaging ability of the system [17].

Although it has been proved that the true random coding photon counting lidar based on Gm-APD has a strong crosstalk suppression ability, and the experiment verifies the ranging imaging ability, References [16-18] do not provide a detailed analysis of the detection performance. This paper analyzes its detection performance in detail. The theory model of correct ranging probability is established based on the photon counting statistics theory and considers the influence of jitter on the correct ranging probability, for the first time. The effects of mean echo photon number, sequence length, mean pulse count density, and pulse width on the correct ranging probability are discussed, and the correctness of the correct ranging probability model is verified by Monte Carlo simulation and experiments. Due to the existence of Poisson distribution noise, the echo intensity of each code is not equal. Therefore, we use the mean echo photon number of multiple codes to represent the echo intensity of the system. The sequence length is determined by the period of the external trigger signal. The number of ' 1 ' code in the true random sequence per second is defined as the density of the ' 1 ' code, which is called the mean pulse count density. The width of the code is defined as the pulse width. The correct ranging probability theoretical model can quantitatively describe the detection performance and provide a solid foundation for further research.

In addition, the correct ranging probability theoretical model provides a theoretical basis for the determination of system parameters such as sequence length, mean pulse count density, single pulse energy, etc. For example, since Gm-APD cannot respond to the echo 
signal intensity, photon counting lidar usually improves the correct ranging probability of the system by increasing the number of accumulated pulses. An excessive increase in the number of accumulated pulses will reduce the detection speed of the system. Based on the established theoretical model, the minimum detection threshold can be determined according to other known parameters (mean pulse count density, single pulse energy, pulse width, noise level (the mean number of noise photons within each code width), etc.) under the premise of ensuring the correct ranging probability. Then, further determine the minimum sequence length to improve the system detection speed.

\section{System Structure and Ranging Principle}

This part introduces the system structure and ranging principle of the true random coding photon counting lidar. In this method, a Gm-APD is used as a random signal generator to generate true nature-based random sequences. More research results can read our previous work, such as the system structure, working principle, and the experimental results of ranging imaging $[17,18]$. Figure 1 is the system structure diagram.

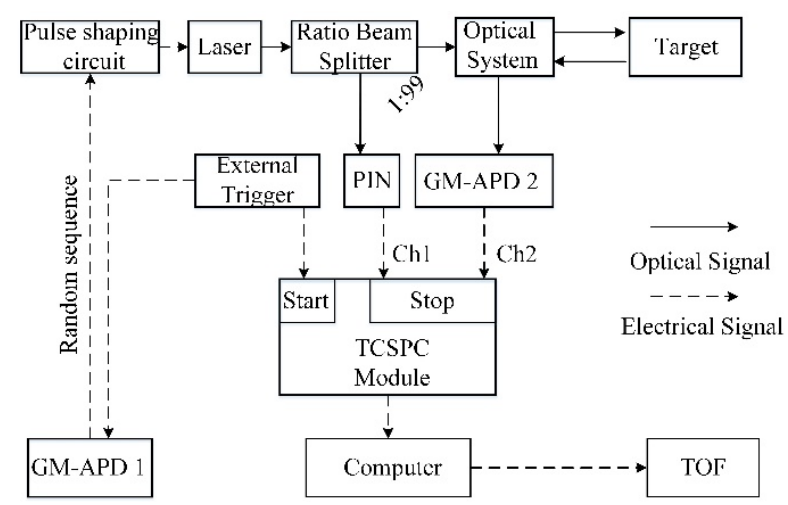

Figure 1. Structure diagram of true random coding photon counting lidar based on Gm-APD.

The system provides timing benchmarks through external trigger sources. We use FPGA as external trigger source to generate periodic trigger signals. If we use the output signal of PIN (P-region, I-region and N-region (PIN)) detector as the trigger signal, the emitted true random sequence will be recorded as discrete pulses because each code element in the true random sequence will become the start signal, resulting in sequence retiming, and cannot guarantee that the whole true random sequence is recorded as a whole. To ensure that all code elements in a sequence have the same timing benchmark, we add an external trigger module to implement this function. The Gm-APD1 continuously generates digital electrical noise, which can be regarded as a true nature-based random sequence. The external trigger signal is equivalent to providing a gating signal for Gm-APD1 to control the sequence length of the true random signal. After pulse shaping circuit, the true random sequence generated by Gm-APD1 directly drives the laser source to obtain the true random optical pulse sequence. The high voltage of the digital electrical noise sequence is recorded as the ' 1 ' code, and the low voltage is recorded as the ' 0 ' code. The true random laser pulses sequence is divided into two parts by a ratio beam splitter: one small part of the energy is detected by a PIN detector, which is used as the transmitted reference signal, recorded as $a(n)$ by TCSPC (time-correlated single photon counting) module, and most of the energy is transmitted to the target. The echo signal is detected by Gm-APD2 and is recorded as $b(n)$ by the TCSPC module by calculating the cross-correlation between the echo signal and the emission template, as shown in Figure 2a [18]. The correlation function is similar to Dirac delta function, $g(\lceil\tau / \Delta t\rceil)=a(n) * b(n)$, where $\Delta t$ is code width. When $\tau$ corresponds to the ToF (Time of Flight), the correlation function $g(\lceil\tau / \Delta t\rceil)$ has max value. The target distance can be determined while unwanted signals from other sources only contribute to noise, as shown in Figure 2b. Code width (pulse width) is the basic matching unit of 
correlation operation. Therefore, the resolution of true random coded photon counting lidar is still determined by the pulse width.

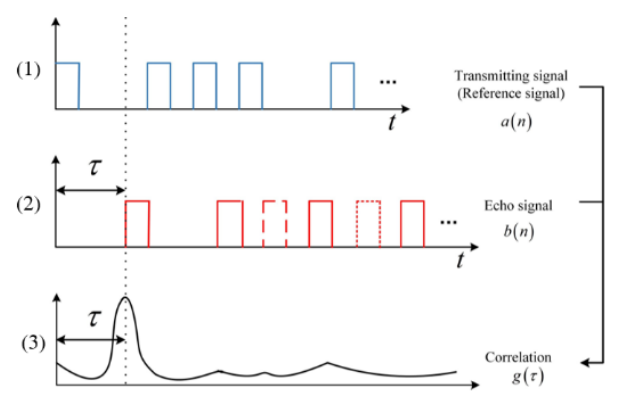

(a)

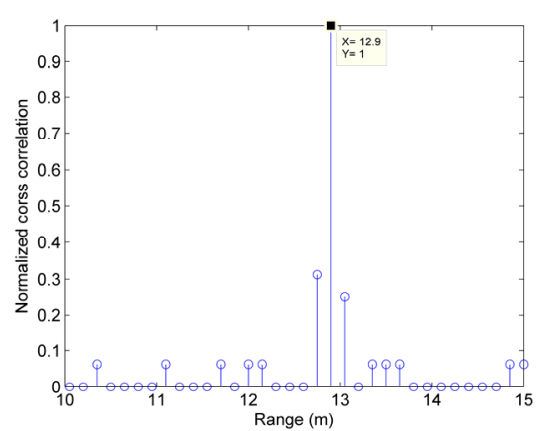

(b)

Figure 2. (a) The ranging principle of true random coding photon counting Lidar. (1) Transmitting signal $a(n)$. (2) Echo Signal $b(n)$. (3) Correlation function $g(\tau)$. (b) Normalized cross correlation.

\section{Theoretical Model of Correct Ranging Probability}

Correct ranging probability is an important index to evaluate the detection performance of photon counting lidar $[17,18]$. The same is true for true random coding photon counting lidar. The correct ranging probability theoretical model can quantitatively evaluate the system performance and provide the basis for the selection of system parameters. Many parameters and definitions are used in this manuscript. For the convenience of reading, the main parameters and definitions are listed in the Table A1.

Since the true random sequence is generated by Gm-APD1, as long as the dead time of Gm-APD1 is not less than Gm-APD2, the ' 1 ' codes in the true random sequence are independent of each other for Gm-APD2 [18]. Since the dead time of Gm-APD is determined by the quenching circuit, the dead time of Gm-APD1 can be longer than that of Gm-APD2 by reasonably designing the quenching circuit. Based on the premise that the ' 1 ' codes are mutually independent, the correct ranging probability model of true random coding photon counting lidar can be established by referring to the pulse accumulation method. The model mainly includes two core parameters: the correct ranging probability of single ' 1 ' code $\left(P_{s n}\right)$ (we define this probability as the counting probability) and the signal recognition threshold $(k)$. The signal recognition threshold $k$ can be considered as the detected codes number in the transmitting true random sequence.

\subsection{Count Probability}

Since the count probability is the same as that of a single pulse, the count probability can be expressed as [24]

$$
P_{s n}=\exp \left(-\int_{t-t_{d 2}}^{t} \psi_{n}(t) d t\right) \cdot\left(1-\exp \left(-\int_{t}^{t+t_{\text {bin }}}\left(\psi_{s}+\psi_{n}\right) d t\right)\right)
$$

where $\psi_{n}(t)$ is mean noise photoelectron flux, $\psi_{s}(t)$ is mean signal photoelectron flux, $t_{d 2}$ is the dead time of Gm-APD2, $t_{b i n}$ is the time bin width. The time bin width is equal to the time resolution of TCSPC module. The bins width affects the timing accuracy of the echo signal.

\subsection{Signal Recognition Threshold}

In the true random coding photon counting lidar system, we define the signal recognition threshold as the minimum detected number of ' 1 ' codes when the target position can be correctly extracted. When the signal recognition threshold is too high, the system will have obvious missing detection. While when the signal recognition threshold is relatively small, the system will have obvious incorrect ranging. However, when the signal recognition threshold is reasonable, it should be in the critical state. 
Firstly, we establish the incorrect ranging probability model of true random coded photon counting lidar. Then, the most reasonable signal recognition threshold can be determined by making the incorrect ranging probability greater than 0 and less than $100 \%$. Assuming that the length of the sequence is $L$ and the average pulse count density of ' 1 ' code in the sequence is $\rho . N=L \cdot \rho$ is transmitted codes number in the sequence. The average pulse interval of each ' 1 ' code is $T_{\rho}=L / N$. The detection probability of noise in each pulse width is $P_{n}=\exp \left(-\int_{t-t_{d 2}}^{t} \psi_{n}(t) d t\right) \cdot\left(1-\exp \left(-\int_{t}^{t+\Delta t} \psi_{n} d t\right)\right)$. For convenience of analysis, the width of the time bin is set to the pulse width, and $n_{t b}=\left\lceil T_{\rho} / \Delta t\right\rceil$ is the number of codes contained in the average pulse interval, where $C_{N}^{i}=\frac{N !}{i !(N-i) !}$. When the threshold value is $k$, the incorrect ranging probability $P_{f a_{k}}$ can be expressed as

$$
P_{f a_{k}}=\sum_{i=k}^{N-k+1} C_{N-k+1}^{i} \cdot n_{t b} \cdot\left(P_{n}\right)^{i}
$$

when incorrect ranging occurs, it means that at least $k$ noise counts have the same arrangement as the pulse sequence that occurred. Therefore, we use the average pulse interval of the transmitted true random sequence as the division unit, divide the entire sequence, then calculate the probability of having the same arrangement as the transmitted sequence, and add up the probability of all noise counts greater than $k$ to obtain the total false alarm probability.

Figure $3 \mathrm{a}, \mathrm{b}$ shows the results of 100 Monte Carlo simulations of the true random coding photon counting lidar. Each line represents the detected codes number at the correlation operation peak position. The hollow point represents the simulation of incorrectly identifying the target position, that is, the case of incorrect ranging. The corresponding ordinate is the detected noise codes number. The solid red dot indicates the correct recognition of the target position. Its corresponding ordinate represents the detected number of ' 1 ' codes.

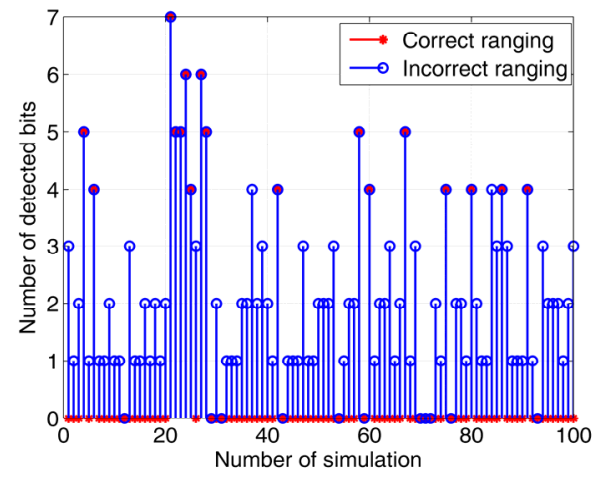

(a)

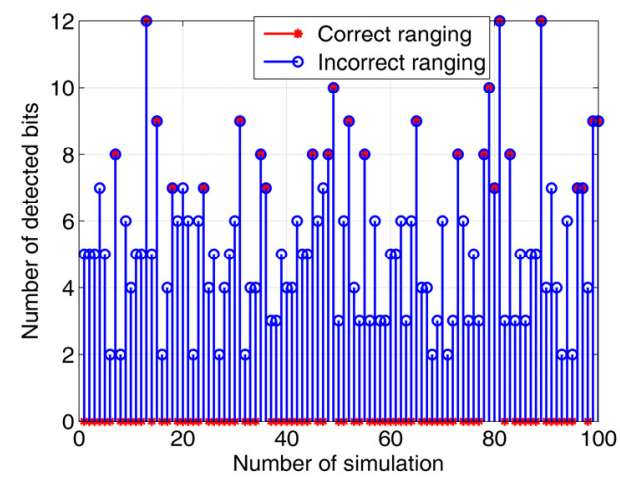

(b)

Figure 3. The detected number of codes at peak position of correlation operation. (a) Sequence length $100 \mu$ s; (b) Sequence length $500 \mu \mathrm{s}$.

The sequence length in Figure $3 a$ is $100 \mu$ s, and the sequence length in Figure $3 b$ is $500 \mu \mathrm{s}$. The mean pulse count density, the mean echo photon number, and the noise count level of the two sequences were 1 Mcps, 0.5, and 1 Mcps, respectively. Taking Figure 3a as an example, when the signal recognition threshold is set to 3 , there will be incorrect ranging detections, and when the signal recognition threshold is set to 5 , there will be many missing detections. For Figure $3 a$, the most reasonable signal recognition threshold should be 4 . There are both correct detections and incorrect detections. That is, the incorrect ranging probability is greater than 0 and less than $100 \%$. Therefore, the most reasonable signal recognition threshold can be determined by calculating the $k$ value which makes the incorrect ranging probability between 0 and 100\%. 


\subsection{Probability of Detected Codes Number Equal to Signal Recognition Threshold}

The number of detected codes (i) equal to the signal recognition threshold $(k)$ can be divided into two cases: the number of detected signal ' 1 ' codes equal to the signal recognition threshold and the number of detected noise codes equal to the signal recognition threshold. For the first case: when the number of detected ' 1 ' codes equals the signal recognition threshold, the target location is not always correctly extracted. There is a chance that noise codes will coincidentally align and equals the threshold, then it will be two correlation peaks, which will cause incorrect detection. For the second case, when the number of detected noise codes is equal to the signal recognition threshold, this detection is certainly incorrect detection. Therefore, it is difficult to directly calculate the correct ranging probability $\left(P_{d_{k}}\right)$ for the first case. However, the incorrect ranging probability $\left(P_{f a_{k}}\right)$ can be analytically expressed by calculating the probability of noise codes. The incorrect ranging probability means that the number of detected noise code elements $\left(i_{n}\right)$ is equal to or greater than the signal recognition threshold, in other words $i_{n} \geq k$. Then, if we know the relationship between the incorrect ranging probability and the correct ranging probability when the detected codes number is equal to the signal recognition threshold, the correct ranging probability can also be quantitatively described.

In order to determine the quantitative relationship between the incorrect ranging probability and correct ranging probability under the signal recognition threshold, we conducted a Monte Carlo simulation. The relationship is simulated under different system parameters (sequence length, mean pulse count density, and noise count level), as shown in Tables 1-3. Sequence length changes from $100 \mu \mathrm{s}: 50 \mu \mathrm{s}: 500 \mu \mathrm{s}$. The results are shown in Table 1 and Figure 4a. The mean pulse count density changes from 0.5 Mcp:0.1 Mcps:2 Mcps. The results are shown in Table 2 and Figure $4 \mathrm{~b}$. The noise count of Gm-APD2 changes from 0.5 Mcps:0.1 Mcps:2 Mcps. The results are shown in Table 3 and Figure 4c. It can be found from Tables 1-3 that according to different system parameters, appropriate signal recognition threshold $k$ should be selected to ensure that the correct detection probability is between 0 and $100 \%$.

Table 1. Correct ranging probability $\left(P_{d_{k}}\right)$ when the detected signal codes number equals the signal recognition threshold $(i=k)$ and incorrect ranging probability $\left(P_{f a_{k}}\right)$ when the detected noise codes number is equal to or greater than the signal recognition threshold $\left(i_{n} \geq k\right)$ under different sequence lengths.

\begin{tabular}{cccccccccc}
\hline & \multicolumn{10}{c}{ Sequence Length } \\
\cline { 2 - 9 } & $\mathbf{1 0 0} \boldsymbol{\mu}$ & $\mathbf{1 5 0} \boldsymbol{\mu s}$ & $\mathbf{2 0 0} \boldsymbol{\mu s}$ & $\mathbf{2 5 0} \boldsymbol{\mu}$ & $\mathbf{3 0 0} \boldsymbol{\mu}$ & $\mathbf{3 5 0} \boldsymbol{\mu s}$ & $\mathbf{4 0 0} \boldsymbol{\mu s}$ & $\mathbf{4 5 0} \boldsymbol{\mu s}$ & $\mathbf{5 0 0} \boldsymbol{\mu s}$ \\
\hline$k$ & 4 & 4 & 5 & 5 & 6 & 6 & 6 & 6 & 7 \\
$P_{f a_{k}}$ & 7.9 & 61.2 & 8.5 & 32.7 & 4.1 & 12.3 & 31.4 & 71.9 & 9.3 \\
$(\%)$ & 66.0 & 12.0 & 70.0 & 21.0 & 82.0 & 51.0 & 25.0 & 7.0 & 71.0 \\
$P_{d_{k}}(\%)$ & $1 \%$ &
\end{tabular}

Table 2. Correct ranging probability $\left(P_{d_{k}}\right)$ when the detected signal codes number equals the signal recognition threshold $(i=k)$ and incorrect ranging probability $\left(P_{f a_{k}}\right)$ when the detected noise codes number is equal to or greater than the signal recognition threshold $\left(I_{n} \geq k\right)$ under different mean pulse count density.

\begin{tabular}{|c|c|c|c|c|c|c|c|c|}
\hline & \multicolumn{8}{|c|}{ Mean Pulse Count Density } \\
\hline & 0.5 Mcps & 0.6 Mcps & 0.7 Mcps & 0.8 Mcps & 0.9 Mcps & 1.0 Mcps & 1.1 Mcps & 1.2 Mcps \\
\hline$k$ & 3 & 3 & 4 & 4 & 4 & 4 & 4 & 4 \\
\hline$P_{f a_{k}}(\%)$ & 50.0 & 87.0 & 1.9 & 3.2 & 5.2 & 7.9 & 11.7 & 16.6 \\
\hline \multirow[t]{3}{*}{$P_{d_{k}}(\%)$} & 0.172 & 0.05 & 0.95 & 0.83 & 0.81 & 0.66 & 59.4 & 51.0 \\
\hline & \multicolumn{8}{|c|}{ Mean Pulse Count Density } \\
\hline & 1.3 Mcps & 1.4 Mcps & 1.5 Mcps & 1.6 Mcps & 1.7 Mcps & 1.8 Mcps & 1.9 Mcps & $2.0 \mathrm{Mcps}$ \\
\hline$k$ & 4 & 4 & 4 & 4 & 5 & 5 & 5 & 5 \\
\hline$P_{f a_{k}}(\%)$ & 22.9 & 30.9 & 40.8 & 52.9 & 1.9 & 2.5 & 3.3 & 4.3 \\
\hline$P_{d_{k}}(\%)$ & 44.0 & 29.0 & 17.0 & 11.0 & 88.4 & 87.0 & 81.6 & 78.0 \\
\hline
\end{tabular}


Table 3. Correct ranging probability $\left(P_{d_{k}}\right)$ when the detected signal codes number equals the signal recognition threshold $(i=k)$ and incorrect ranging probability $\left(P_{f a_{k}}\right)$ when the detected noise codes number is equal to or greater than the signal recognition threshold $\left(i_{n} \geq k\right)$ under different mean noise count density.

\begin{tabular}{|c|c|c|c|c|c|c|c|c|}
\hline & \multicolumn{8}{|c|}{ Mean Noise Count Level } \\
\hline & 0.5 Mcps & 0.6 Mcps & 0.7 Mcps & 0.8 Mcps & 0.9 Mcps & 1.0 Mcps & 1.1 Mcps & 1.2 Mcps \\
\hline$k$ & 3 & 3 & 4 & 4 & 4 & 4 & 4 & 4 \\
\hline$P_{f a_{k}}(\%)$ & 0.5104 & 0.882 & 0.019 & 0.0324 & 0.052 & 0.079 & 0.116 & 0.164 \\
\hline \multirow[t]{3}{*}{$P_{d_{k}}(\%)$} & 0.08 & 0.02 & 0.91 & 0.88 & 0.81 & 0.66 & 0.61 & 0.48 \\
\hline & \multicolumn{8}{|c|}{ Mean Noise Count Level } \\
\hline & 1.3 Mcps & 1.4 Mcps & 1.5 Mcps & 1.6 Mcps & 1.7 Mcps & 1.8 Mcps & 1.9 Mcps & $2.0 \mathrm{Mcps}$ \\
\hline$k$ & 4 & 4 & 4 & 4 & 4 & 5 & 5 & 5 \\
\hline$P_{f a_{k}}(\%)$ & 22.6 & 30.4 & 40.1 & 51.9 & 66.2 & 2.4 & 3.1 & 4.1 \\
\hline$P_{d_{k}}(\%)$ & 38.9 & 34.8 & 29.9 & 18.1 & 11.2 & 91.4 & 88.1 & 83.0 \\
\hline
\end{tabular}

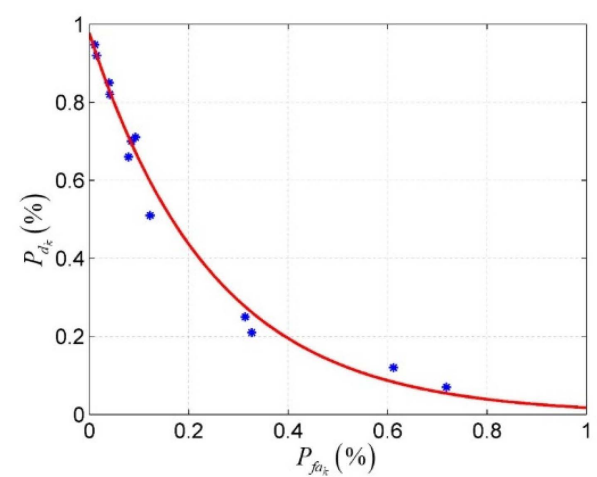

(a)

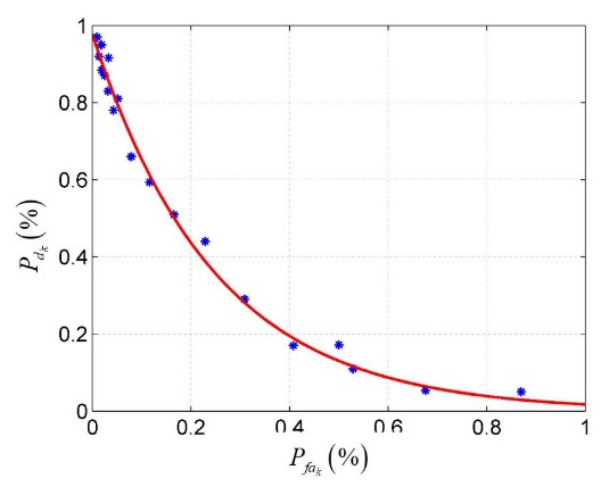

(b)

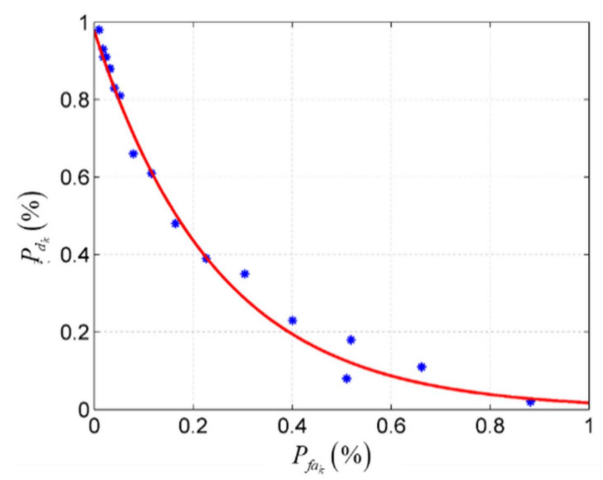

(c)

Figure 4. Correct ranging probability $\left(P_{d_{k}}\right)$ when the detected signal codes number equals the signal recognition threshold and incorrect ranging probability $\left(P_{f a_{k}}\right)$ when the detected noise codes number is equal to or greater than the signal recognition threshold under different system parameters. (a) Under different sequence length; (b) Under different pulse count density; (c) Under different noise count level.

It can be found from Table 1 that the longer the sequence, the greater the signal recognition threshold. When the signal recognition threshold is the same, the longer the sequence is, the higher the incorrect ranging probability of the system is, and the lower the correct ranging probability is. It can be found from Tables 2 and 3 that the mean pulse count density and noise count level also conform to the same law.

It can be found from Tables 1-3 and Figure 5 that under different system parameters, the changes of the relationship between the correct ranging probability $P_{d_{k}}$ and the incorrect ranging probability $P_{f a_{k}}$ have a consistent trend. In other words, the influences of the three 
system parameters on the relationship between correct ranging probability $P_{d_{k}}$ and incorrect ranging probability $P_{f a_{k}}$ are not significantly different. Based on this premise, in order to obtain more accurate and more general fitting result, we merge all data under different sequence length, pulse counting density and noise level. The curve fitting results are shown in Figure 5.

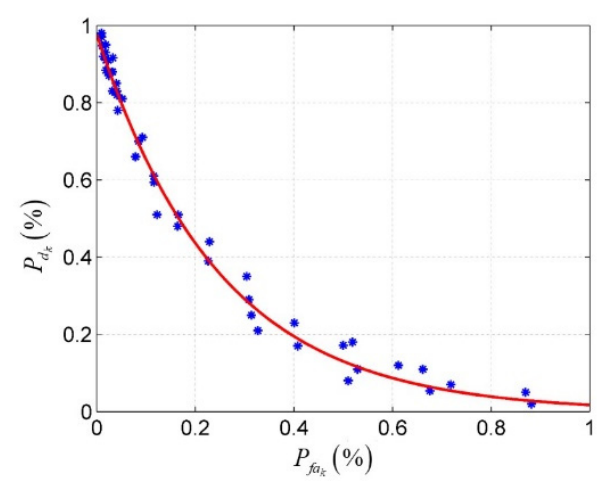

Figure 5. The relationship between $P_{f a_{k}}$ and $P_{d_{k}}$.

In order to quantitatively describe the relationship between incorrect ranging probability $P_{f a_{k}}$ and correct ranging probability $P_{d_{k}}$, curve fitting is carried out on the discrete data in Figure 4. The result of the curve fitting is shown in Equation (3)

$$
P_{d_{k}}=0.9775 \cdot \exp \left(-4.0306 \cdot P_{f a_{k}}\right)
$$

The red solid line in Figure 4 is the theoretical result of Equation (3), and the blue discrete point is composed of the simulation data in Figure 3. It can be found that the fitting result has a high correlation with the simulation data. At the same time, we use Equation (3) to fit the simulation data under different system parameters in Figure 3, and it can be found that the discrete points under three groups of simulation data are highly consistent with the theoretical results.

\subsection{Correct Ranging Probability Model without Considering System Jitter}

Under reasonable signal recognition threshold conditions, the correct ranging probability $P_{D}$ of true random coding photon counting lidar can be written as

$$
P_{D}(k \leq N)=P_{D}(i=k)+P_{D}(k<i \leq N)
$$

where $k$ is the signal recognition threshold, and $i$ is the number of correctly detected codes. It can be found from Figure 2 that the correct ranging probability $P_{D}$ of the true random coding photon counting lidar is composed of two parts: the correct ranging probability $P_{d_{k}}$ that the detected signal ' 1 ' codes number is equal to the signal recognition threshold (corresponding to the first item in Equation (4)) and the correct ranging probability that the detected signal ' 1 ' codes number is higher than the signal recognition threshold (corresponding to the second item in Equation (4)). When the detected ' 1 ' code number is equal to the signal recognition threshold, the probability of correctly extracting the target position can be expressed as Equation (2). Then, Equation (4) can be further written as

$$
P_{D}(k \leq N)=C_{N}^{i} P_{s n}^{i}\left(1-P_{s n}\right)^{N-i} \cdot P_{d_{k}}+\sum_{i=k+1}^{N} C_{N}^{i} P_{s n}^{i}\left(1-P_{s n}\right)^{N-i} \cdot P_{d_{k}}
$$

where $C_{N}^{i}=\frac{N !}{i !(N-i) !}$. The first term in Equation (5) is composed of two parts: the first part represents the probability of just detecting $k$ ' 1 ' codes, and the second part represents the probability of correctly extracting the target position when the sequence detects $k$ ' 1 ' codes. The product of the two parts is used to represent the probability that the target 
can be correctly extracted when the detected ' 1 ' codes is equal to the signal identification threshold $k$. The second term in Equation (5) is similar to the first term, which represents the probability that the sequence can correctly extract the target position when the detected ' 1 ' codes number is higher than the signal recognition threshold. Eventually, based on Equation (5), we have established the correct ranging probability theoretical model of truly random coding photon counting lidar without considering system jitter.

\subsection{Correct Ranging Probability Model with Considering System Jitter}

When there is a large jitter in the system, the impact of the jitter on the correct ranging probability should be considered. The reason why the jitter affects the correct ranging probability is that the true random coding photon counting lidar is based on the correlation operation to calculate the target distance. The jitter causes the ' 1 ' code to shift forward or backward, resulting in matching error, thereby impacting the correct ranging probability. In the true random coding photon counting lidar system, the jitter is mainly composed of laser source, Gm-APD2 and TCSPC. In order to facilitate the analysis, the three jitter errors are considered comprehensively, and the FWHM (Full Width at Half Maxima) of the total jitter is $\Delta_{t \text { Jitter }}=\sqrt{\Delta_{t}^{2}{ }_{\text {Laser }}+\Delta_{t}^{2} t_{\text {Det }}+\Delta_{t}^{2} t_{\text {Tsps }}}$.

When considering the effect of system jitter, the Equations (2)-(5) needs to be modified. Firstly, modify the incorrect ranging probability formula (Equation (2)). At this time, the incorrect ranging will include two parts: (1) the incorrect ranging caused by noise and (2) the incorrect ranging caused by the misplaced ' 1 ' code due to the jitter. $\Delta t_{\text {jitter }}$ is the FWHM of the system jitter, and the standard deviation of the jitter is $\sigma_{t j i t t e r}=\Delta_{t j i t t e r} / 2.355$. Assuming that the jitter obeys the normal distribution of standard deviation $\sigma_{t j i t t e r}$ and mean value 0 , then its probability distribution function is as follows:

$$
F(t)=\frac{1}{\sqrt{2 \pi} \sigma_{t J i t t e r}} \int_{-\infty}^{t} e^{-\frac{t^{2}}{2 \sigma_{t j i t t e r}^{2}}} d t-\infty<t<\infty
$$

When the jitter is considered, the probability that the ' 1 ' code is not misaligned is

$$
P_{s n_{-} \text {Right }}=P_{s n} \cdot\left(F\left(\frac{\Delta t}{2}\right)-F\left(-\frac{\Delta t}{2}\right)\right)
$$

However, the probability of jitter dislocation of ' 1 ' code is complex, which needs to be considered in two parts. Step 1: determine that the ' 1 ' code can jitter up to a few pulse widths, in other words, there are several jitter positions for this code:

$$
M=2\left\lfloor\frac{\left(3 \cdot \sigma_{t J i t t e r}-\Delta t / 2\right)}{\Delta t}\right\rfloor
$$

Step 2: determine the probability of jitter occurring at each position:

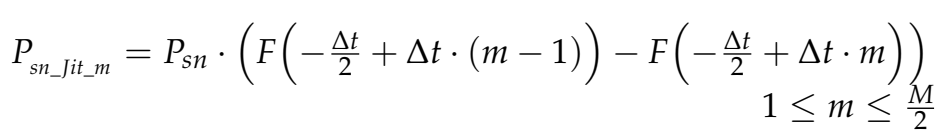

Therefore, when considering the system jitter, the incorrect ranging probability equation (Equation (2)) can be written as:

$$
\begin{aligned}
& p_{f a_{k-} J i t}=\sum_{i=k}^{N-k+1} n_{t b} \cdot\left(P_{n}\right)^{i} \cdot C_{N-k+1}^{i}+2 \cdot \sum_{m=1}^{M / 2} C_{N}^{i} P_{s n_{-} j i t_{-} m}^{i}\left(1-P_{s n_{-} \text {Jit_m }}\right)^{N-i} \\
& -2 \cdot\left(\sum_{i=k}^{N-k+1} n_{t b} \cdot\left(P_{n}\right)^{i} \cdot C_{N-k+1}^{i}\right) \cdot\left(\sum_{m=1}^{M / 2} C_{N}^{i} P_{s n_{-}{ }_{i} \_m}^{i}\left(1-P_{s n_{-} j i t_{-} m}\right)^{N-i}\right) \\
& -\left(\sum_{m=1}^{M / 2} C_{N}^{i} P_{s n_{-} i t_{\_} m}^{i}\left(1-P_{s n_{-} i t t_{-} m}\right)^{N-i}\right)^{2}\left(0 \leq P_{f a_{k}} \leq 1\right)
\end{aligned}
$$


The correct ranging probability formula (Equation (5)) of reasonable threshold can be adjusted as:

$$
P_{d_{k-} J i t}=0.9775 \cdot \exp \left(-4.0306 \cdot P_{f a_{k-} J i t}\right)
$$

Secondly, when considering the jitter, the correct ranging probability of the true random coding photon counting lidar can be modified as

$$
\begin{aligned}
P_{D_{-} J i t}(k \leq N)= & \sum_{i=k+1}^{N} C_{N}^{i} P_{s n \_ \text {Right }}^{i}\left(1-P_{\text {sn_Right }}\right)^{N-i} \\
& +C_{N}^{k} P_{s n \_R i g h t}^{k}\left(1-P_{s n \_R i g h t}\right)^{N-k} \cdot P_{d_{k-} J i t}
\end{aligned}
$$

\section{Verify the Correct Ranging Probability Theoretical Model with Monte Carlo Simulation}

\subsection{Verify the Correct Ranging Probability Model without Considering System Jitter}

The main factors affecting the correct ranging probability of true random coding photon counting lidar are mean echo photon number, mean pulse count density, sequence length, pulse width, and mean noise count level. In order to prove the correctness of the correct ranging probability theoretical model, a Monte Carlo simulation is used to verify the influence of these factors on correct ranging probability one by one, and the simulation results are compared with the theoretical results obtained by Equation (5). According to the system parameters, random numbers are generated according to Poisson distribution, and the influence of detector dead time is considered. The echo sequence generated by simulation is correlated with the reference sequence, and the peak position of the correlation operation is taken as the target position. Five thousand simulations are completed under each system parameter. The target position in simulation is known, so the target position determined by simulation can be compared with the real target position to determine the correct ranging probability. The simulation results are compared with the theoretical results obtained by Equation (5).

The circle represents the simulation result, and the solid line represents the theoretical result. Different system parameters are distinguished with different colors. The mean echo photon number is the core factor that affects the correct ranging probability. Therefore, the theoretical analysis and Monte Carlo simulation of the correct ranging probability are completed with a different mean echo photons number as the main variable parameter. The mean echo photon number in the simulation refers to the mean echo photons number per code, assuming that the detection efficiency of Gm-APD2 is $2 \%$; when the mean echo photons number is 1 , the mean photoelectron number generated by each code is about 0.02 .

From Figure $6 a-d$, it can be found that as the mean echo photons number increases, the correct ranging probability of the sequence gradually increases and tends to be saturated. It can be found from Figure 6a that when the correct ranging probability is not saturated, the higher the mean pulse count density, the higher the correct ranging probability under the same echo photon number. From Figure $6 b$, it can be found that the sequence length has the same influence on the correct ranging probability. Figure $6 \mathrm{c}, \mathrm{d}$ shows the influence of pulse width and average noise count level on the correct ranging probability. It can be found that the smaller the pulse width, the higher the correct ranging probability. In our method, we take the code as the analysis unit. Therefore, in the case of the same number of echo photons per code, the shorter the code, the smaller the noise count of each code. Therefore, it has higher correct detection probability. Meanwhile, the smaller the mean noise count level, the higher the correct ranging probability. 


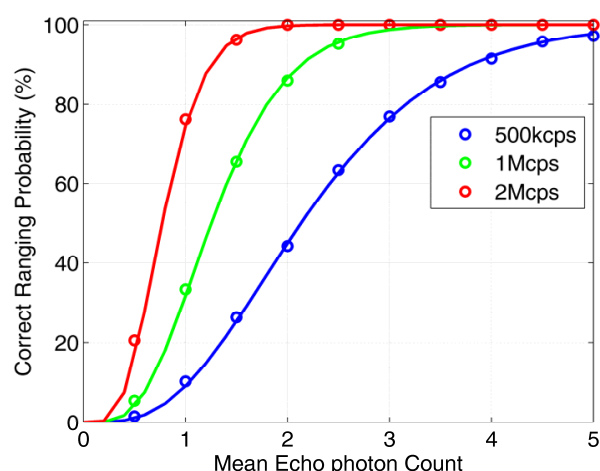

(a)

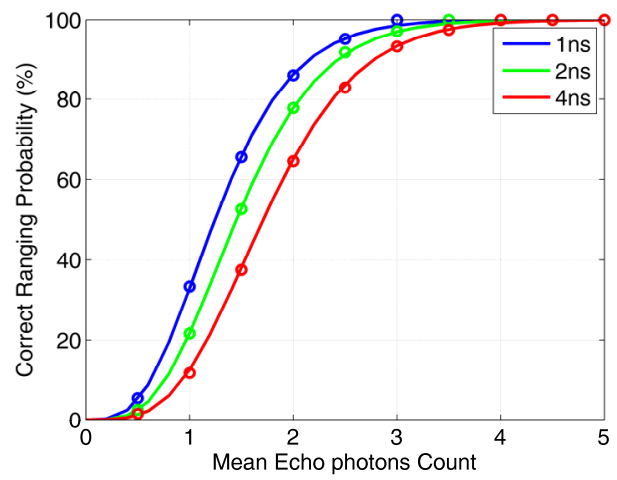

(c)

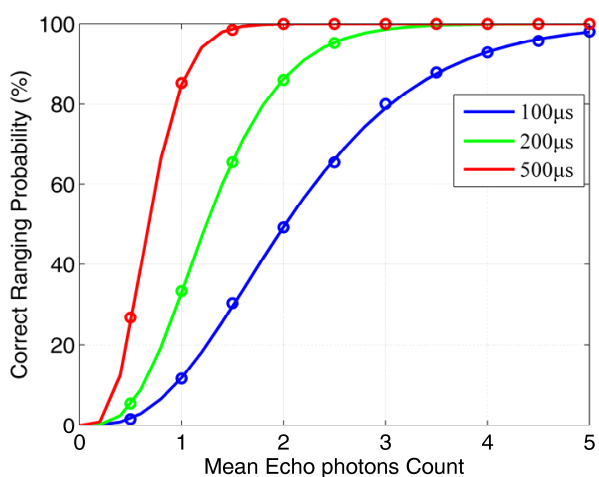

(b)

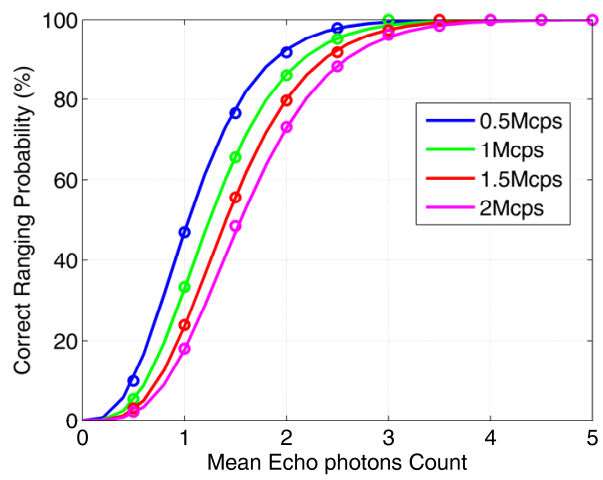

(d)

Figure 6. Monte Carlo simulation verification of correct ranging probability: (a) the influence of mean echo photon number and mean pulse count density on correct ranging probability (sequence length $200 \mu$ s, pulse width 1ns, mean noise count density $1 \mathrm{Mcps}$ ); (b) the influence of sequence length on correct ranging probability (pulse count 1 Mcps, noise count density 1 Mcps, pulse width $1 \mathrm{~ns}$ ); (c) the influence of pulse width on correct ranging probability (sequence length $200 \mu \mathrm{s}$, mean pulse count $1 \mathrm{Mcps}$ ); (d) the effect of noise count density on detection probability (sequence length $200 \mu \mathrm{s}$, mean pulse count density 1 Mcps, pulse width $1 \mathrm{~ns}$ ).

\subsection{Verify the Correct Ranging Probability Model with Considering System Jitter}

Figure 7 shows the variation of correct ranging probability with jitter under different mean echo photon number, when the sequence length is $100 \mu \mathrm{s}$, the mean pulse count density is $1 \mathrm{Mcps}$, the mean noise count density is $1 \mathrm{Mcps}$, and the pulse width is $1 \mathrm{~ns}$. When the FWHM of system jitter is approximately less than half of the pulse width $(0.2 \cdot 2.355=$ $0.471 \mathrm{~ns})$, jitter has little effect on the correct ranging probability, but with the increase of jitter, the impact of jitter on correct ranging probability will become extremely significant. It can be seen from Figure 7 that when the jitter is larger than half of the pulse width, the correct ranging probability decreases approximately linearly. In the case of a different mean echo photon number, there is a slight difference in the effect of the jitter on the correct ranging probability. When the mean echo photon number is small, the increase of jitter has little impact on the correct ranging probability. This is because when the number of echo photons is small, the false alarm caused by the jitter of the ' 1 ' code is low. In other words, the incorrect detection generated in the second part of the Equation (11) is small, which slows down the downward trend of correct ranging probability to a certain extent. 


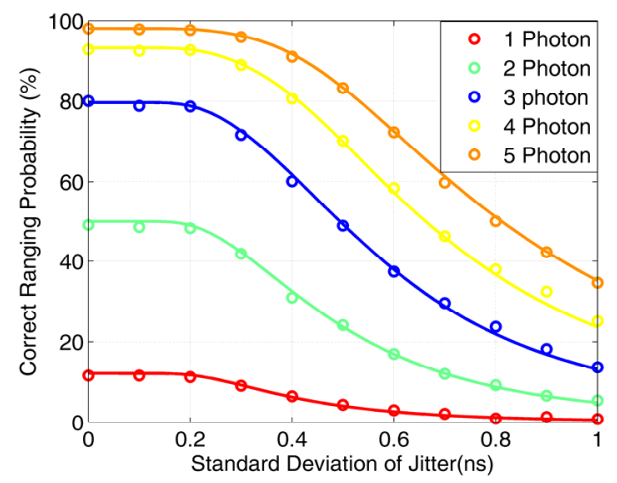

Figure 7. The influence of jitter on correct ranging probability under different echo photons number.

Figure 8 shows the effect of system jitter on the correct ranging probability under different pulse widths. It shows that the correct ranging probability varies with jitter when the pulse width is $1 \mathrm{~ns}, 2 \mathrm{~ns}$, and $4 \mathrm{~ns}$, respectively; when the sequence length is $100 \mu \mathrm{s}$, the mean pulse count density is $1 \mathrm{Mcps}$, the mean noise count density is $1 \mathrm{Mcps}$, and the mean echo photon number is about 3 . It can be found that under different pulse widths, the impact of jitter on the correct ranging probability mainly has two obvious phenomena. First, when the jitter is less than a certain value, jitter almost has no effect on the correct ranging probability. The three different pulse widths satisfy this rule, and the wider the code is, the larger the corresponding value is. Second, we can find that when the jitter exceeds a certain value, with the increase of jitter, the red curve decreases obviously, the green curve takes the second place, and the blue curve is the least obvious; that is to say, the wider the code, the stronger the ability of anti-jitter.

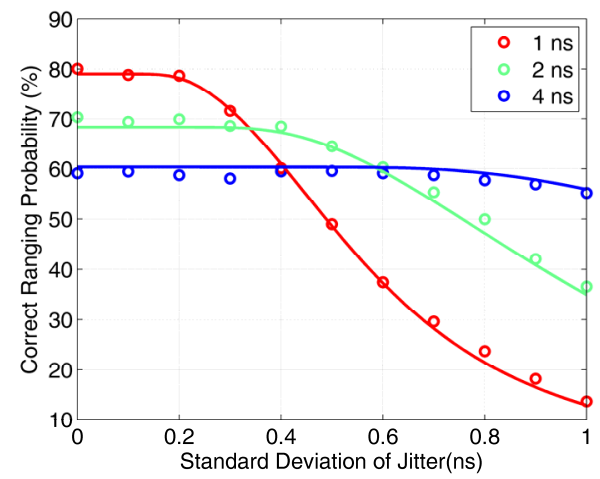

Figure 8. The influence of jitter on correct ranging probability under different pulse widths.

It can be found from Figures 6-8 that the theoretical results are in high agreement with the simulation results. This proves that the correct ranging probability model is correct regardless of whether the impact of jitter is considered. Overall, we first prove the correctness of the theoretical model of correct ranging probability based on a Monte Carlo simulation. In the Section 5, we will further verify the correctness of the theoretical model through experiments.

\section{Verify the Correct Ranging Probability Theoretical Model with Experiment}

In order to further verify the theoretical model, we have built an experimental platform for a lidar system as shown in Figure 9a. The main experimental parameters are shown in Table 4 . For the true random coding photon counting lidar, system jitter is mainly composed of three jitter sources: laser source, Gm-APD2 and TCSPC module. The jitter of Gm-APD2 can be obtained by datasheet, and the jitter of the laser source and TCSPC module can be measured by high bandwidth signal generator and high bandwidth linear detector. We adopt a simpler method to determine the system jitter. Instrument Response Function 
(IRF) summarizes overall characteristics of pulse width and system jitter. The FWHM (Full Width at Half Maxima) of the IRF $=\sqrt{n_{p}^{2}+\Delta_{t J i t t e r}^{2}}$, where $n_{p}$ is pulse width and $\Delta_{t J i t t e r}$ is system jitter. For a fixed pulse width and system jitter, we can determine the system jitter by measuring the system response function. The IRF is obtained by measuring a flat target. Through a large number of statistical experiments, the IRF is shown in the Figure 9b. Because the FWHM of IRF is 1.069 ns, pulse width is 1ns, so the FWHM of system jitter is 441 ps. Because the FWHM of system jitter is less than half of the minimum pulse width, the jitter has little effect on the correct ranging probability of our system. Therefore, the experiment verifies the correct ranging probability model without considering system jitter. The target with known distance is detected in the experiment. By comparing the distance detected by the experiment with the real distance, the correct detection probability of the system is determined.

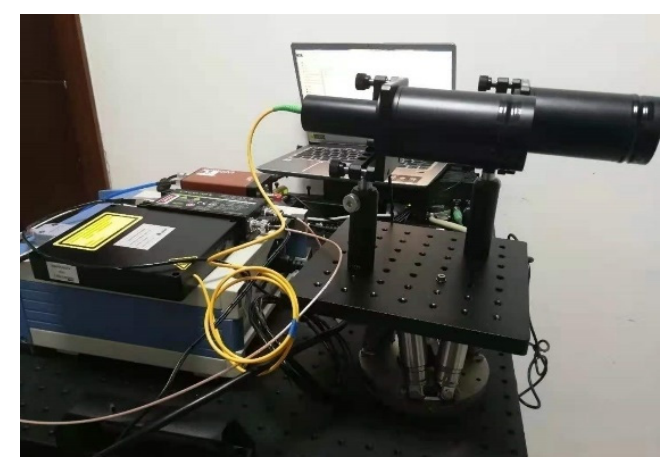

(a)

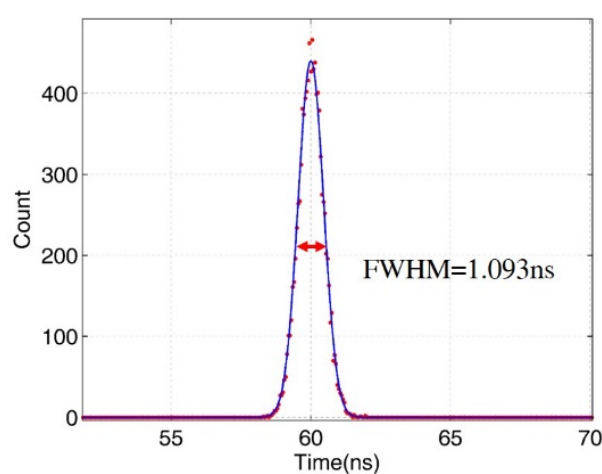

(b)

Figure 9. (a) True random coding photon counting lidar experimental platform. (b) IRF signal detections with Gaussian curve fitting, where estimated Gaussian RMS width is 0.464 ns, thus FWHM is 1.09 ns.

Table 4. System parameters for true random coding photon counting lidar.

\begin{tabular}{cc}
\hline Parameter & Value \\
\hline Wavelength & $1064 \mathrm{~nm}$ \\
Dead time & $45 \mathrm{~ns}$ (Gm-APD1) \\
Pulse width & $/ 25 \mathrm{~ns}$ (GM-APD2) \\
Sequence length & $1 / 2 / 4 \mathrm{~ns}$ \\
Mean noise count density & $100 / 200 / 500 \mu \mathrm{s}$ \\
Mean echo photon number & $1 \mathrm{Mcps}$ \\
Mean pulse count density & $1: 0.5: 5$ \\
Detection efficiency (Gm-APD2) & $0.5 / 1 / 2 \mathrm{Mcps}$ \\
Time jitter (FWHM/Gm-APD2) & $2 \%$ \\
\end{tabular}

Figure $10 \mathrm{a}-\mathrm{c}$ shows the variation of correct ranging probability with mean echo photon number under different sequence lengths, different pulse count density and different pulse width, respectively. The average detection probability of the emitted ' 1 ' code is calculated, and then the mean echo photon number of ' 1 ' codes is determined by the detection probability model of ' 1 ' code. The circle represents the experimental result, and the solid line represents the theoretical result. Distinguish different system parameters with different colors. From Figure 10 we can find that the experimental results are highly consistent with the theoretical results. Therefore, we have verified the correctness of the theoretical model through experiments. Based on the established theoretical model, the correct ranging probability can be effectively estimated according to the system parameters, which provides a reliable means for evaluating the detection performance of the system. 


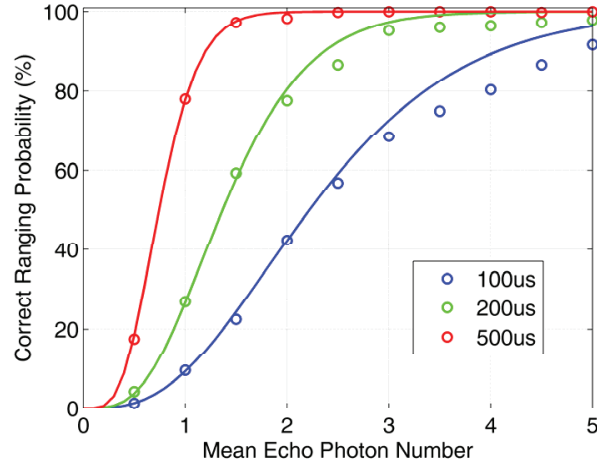

(a)

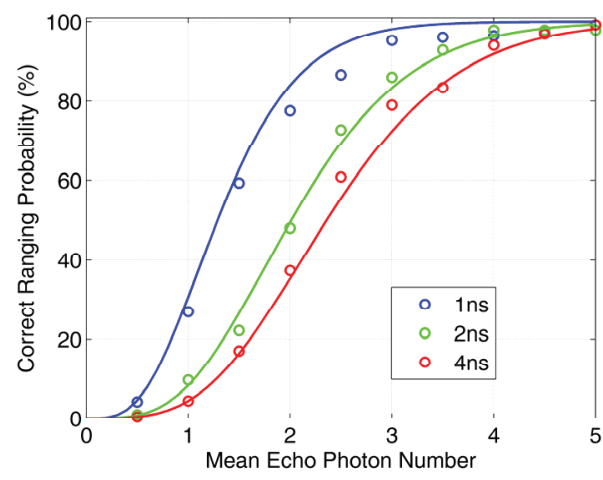

(c)

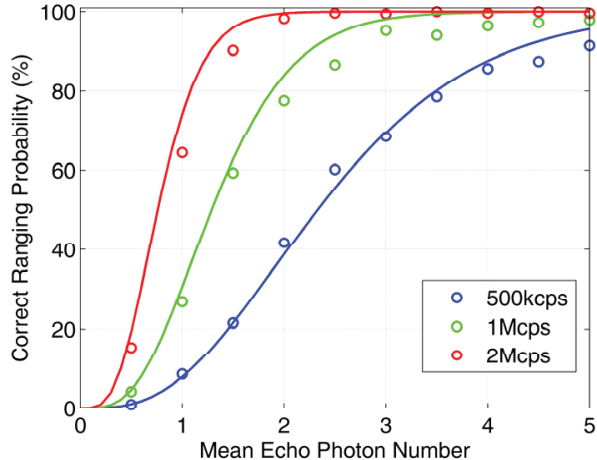

(b)

Figure 10. Experimental verification of correct ranging probability under different system parameters: (a) the influence of sequence length on correct ranging probability (mean pulse count density 1 Mcps, pulse width 1 ns, mean noise count density $1 \mathrm{Mcps}$ ), (b) the influence of mean pulse count on correct ranging probability (sequence length $200 \mu$ s, pulse width $1 \mathrm{~ns}$, mean noise count density $1 \mathrm{Mcps}$ ), (c) the influence of pulse width on correct ranging probability (sequence length $200 \mu \mathrm{s}$, mean pulse count density 1 Mcps, mean noise count density 1 Mcps).

The echo intensity detected by photon counting lidar is in a photon level. Such high detection sensitivity makes it very sensitive to small changes in the system. Through our simulation and experiment, we can find that the change of one photon may make the detection probability change more than $50 \%$. In the actual system, due to laser source instability, background noise changes, number of statistics, and other factors will cause changes in detection probability. As far as our experiment is concerned, there are two main causes of error: (1) the change of echo intensity caused by laser source instability and, (2) in the experiment, it is necessary to accurately adjust the echo photon to a certain intensity, such as 2 echo photons, which is very difficult for practical operation. Therefore, due to many inevitable errors in the experimental process, there are certain errors between the experimental and theoretical results. The simulation system can avoid these errors, so the simulation results are highly consistent with the theoretical results.

\section{Discussion}

The main contribution of this paper is to establish a theoretical model of the correct ranging probability for this true random coding photon counting lidar detection method. Based on the theoretical model, the correct ranging probability can be effectively estimated according to the system parameters, which provides a reliable means for evaluating the detection performance of the system. To know more about this method working principle, system imaging performance analysis, you can read our previous articles in detail $[17,18]$. It should be pointed out that the experimental results in Figure 10a,b have been shown in Reference [18], but the analysis of the theoretical model is added in this paper. The experimental results are mainly to verify the correctness of the theoretical model. 


\section{Conclusions}

In the future, with the intensive application of lidar, higher requirements will be put forward for the anti-interference capability of lidar systems. Almost at the same time, Tsai and we proposed to use Gm-APD as a random signal generator and use the true random sequence generated by Gm-APD to modulate the laser source to improve the system's anti-crosstalk ability. Previous studies have verified the anti-crosstalk capability, ranging and imaging capabilities but did not involve analysis of the correct ranging probability of this method. In this paper, the parameters that affect the correct ranging probability are analyzed in detail, and the theoretical model of correct ranging probability is established. In this model, the influence of system jitter on correct ranging probability is considered for the first time. The correctness of the model is verified by simulation and experiment. The correct ranging probability model provides an effective means for the detection performance evaluation and fills an important link for the establishment of the complete theoretical framework of the true random coding photon counting lidar system.

Author Contributions: Conceptualization, Y.Y. and Z.W.; funding acquisition, C.C.; methodology, Y.Y. and K.M.; software, Y.Y.; supervision, B.X.; validation, X.L.; visualization, Y.Y.; writing-original draft, Y.Y.; Writing-review and editing, X.W., F.Z., X.P., Q.Z., and H.L. All authors have read and agreed to the published version of the manuscript.

Funding: This research is jointly supported by the following project grants: Natural Science Foundation of Shandong Province (ZR2021QF055, ZR2021QF015); Talent Induction Doubling Plan QLUT (2021YY01024, 2021YY01017); Key Research and Development Plan of Shandong Province (2020CXGC010104); GuangDong Basic and Applied Basic Research Foundation (2020B1515120056); Basic Research Enhancement project SCSC (2021JC02004).

Institutional Review Board Statement: Exclude this statement.

Informed Consent Statement: Exclude this statement.

Data Availability Statement: Exclude this statement.

Conflicts of Interest: The authors declare no conflict of interest.

\section{Appendix A}

For the convenience of reading, the parameters and definitions are listed in the Table A1.

Table A1. List of parameters and definitions.

\begin{tabular}{|c|c|c|c|}
\hline$P_{s n}$ & $\begin{array}{l}\text { correct ranging probability of single ' } 1 \text { ' } \\
\text { code }\end{array}$ & $\psi_{n}(t)$ & mean noise photoelectron flux \\
\hline$P_{n}$ & $\begin{array}{l}\text { detection probability of noise in each pulse } \\
\text { width }\end{array}$ & $\psi_{s}(t)$ & mean signal photoelectron flux \\
\hline$P_{f a_{k}}$ & incorrect ranging probability & $t_{d 2}$ & dead time of Gm-APD2 \\
\hline$P_{d_{k}}$ & correct ranging probability & $t_{\text {bin }}$ & time bin width \\
\hline$k$ & signal recognition threshold & $\Delta t_{\text {Jitter }}$ & the FWHM of the system jitter \\
\hline$L$ & sequence length & $N$ & $\begin{array}{l}\text { transmitted codes number in the } \\
\text { sequence. }\end{array}$ \\
\hline$\rho$ & mean pulse count density of ' 1 ' code & $T_{\rho}$ & average pulse interval of each ' 1 ' code \\
\hline$P_{d_{k}}$ & \multicolumn{3}{|c|}{ correct ranging probability when the detected signal codes number equals the signal recognition threshold } \\
\hline$P_{f a_{k}}$ & \multicolumn{3}{|c|}{$\begin{array}{l}\text { incorrect ranging probability when the detected noise codes number is equal to or greater than the signal } \\
\text { recognition threshold }\end{array}$} \\
\hline$P_{D}$ & \multicolumn{3}{|c|}{ the correct ranging probability of lidar system } \\
\hline
\end{tabular}




\section{References}

1. Steindorfer, M.A.; Kirchner, G.; Koidl, F.; Wang, P.; Jilete, B.; Flohrer, T. Daylight space debris laser ranging. Nat. Commun. 2020, 11, 1-6. [CrossRef] [PubMed]

2. Li, Z.; Ye, J.; Huang, X.; Jiang, P.-Y.; Cao, Y.; Hong, Y.; Yu, C.; Zhang, J.; Zhang, Q.; Peng, C.-Z.; et al. Single-photon imaging over $200 \mathrm{~km}$. Optica 2021, 8, 344-349. [CrossRef]

3. Degnan, J.J. Scanning, Multibeam, Single Photon Lidars for Rapid, Large Scale, High Resolution, Topographic and Bathymetric Mapping. Remote Sens. 2016, 8, 958. [CrossRef]

4. Prochazka, I.; Kodet, J.; Blazej, J.; Kirchner, G.; Koidl, F. Photon counting detector for space debris laser tracking and lunar laser ranging. Adv. Space. Res. 2014, 54, 755-758. [CrossRef]

5. Markus, T.; Neumann, T.; Martino, A.; Abdalati, W.; Brunt, K.; Csatho, B.; Farrell, S.; Fricker, H.; Gardner, A.; Harding, D.; et al. The Ice, Cloud, and land Elevation Satellite-2 (ICESat-2): Science requirements, concept, and implementation. Remote Sens. Environ. 2017, 190, 260-273. [CrossRef]

6. McCarthy, A.; Collins, R.J.; Krichel, N.J.; Fernández, V.; Wallace, A.M.; Buller, G.S. Long-range time-of-flight scanning sensor based on high-speed time-correlated single-photon counting. Appl. Opt. 2009, 48, 6241-6251. [CrossRef] [PubMed]

7. Petit, J.; Stottelaar, B.; Feiri, M.; Kargl, F. Remote attacks on automated vehicles sensors: Experiments on camera and lidar. Black Hat Eur. 2015, 11, 995.

8. Matthey, R.; Mitev, V. Pseudo-random noise-continuous-wave laser radar for surface and cloud measurements. Opt. Laser Eng. 2005, 43, 557-571. [CrossRef]

9. Du, P.; Geng, D.; Wang, W.; Gong, M. Laser detection of remote targets applying chaotic pulse position modulation. Opt. Eng. 2015, 54, 114102. [CrossRef]

10. González, E.; Navarro, S.A.; Pérez Serrano, A.; Vilera, M.; Tijero, J.M.G.; Esquivias, I. Theoretical Analysis of Random-Modulation Continuous Wave LIDAR. In Proceedings of the 9 a Reunión Española de Optoelectrónica (OPTOEL'15), Salamanca, Spain, 13-15 July 2015; pp. 1-5.

11. Zhang, F.; Du, P.; Liu, Q.; Gong, M.; Fu, X. Adaptive strategy for CPPM single-photon collision avoidance LIDAR against dynamic crosstalk. Opt. Express 2017, 25, 12237-12250. [CrossRef] [PubMed]

12. Cheng, C.H.; Chen, C.Y.; Chen, J.D.; Pan, D.K.; Ting, K.T.; Lin, F.Y. 3d pulsed chaos lidar system. Opt. Express 2018, 26, $12230-12241$.

13. Hao, J.; Gong, M.-L.; Du, P.-F.; Lu, B.-J.; Zhang, F.; Zhang, H.-T.; Fu, X. Ultra-low power anti-crosstalk collision avoidance light detection and ranging using chaotic pulse position modulation approach. Chin. Phys. B 2016, 25, 250-257. [CrossRef]

14. Hao, J.; Gong, M.; Du, P.; Lu, B.; Zhang, F.; Zhang, H.; Fu, X. A Novel CPPM Anti-Crosstalk Collision Avoidance Lidar with Ultra-Low Laser Power. In Proceedings of the 8th International Symposium on Advanced Optical Manufacturing and Testing Technologies: Optoelectronic Materials and Devices, Suzhou, China, 26-29 April 2016; International Society for Optics and Photonics: Bellingham, WA, USA, 2016; Volume 9686, pp. 968607-968616.

15. Wang, B.; Qian, J.; Zhao, T.; Wang, Y. Anti-jamming performance of chaotic lidar. Chin. J. Lasers 2011, 38, 0514002. [CrossRef]

16. Tsai, C.; Liu, Y. Anti-Interference Single-Photon LiDAR Using Stochastic Pulse Position Modulation. Opt. Lett. 2020, 45, 439-442. [CrossRef]

17. Liu, B.; Yu, Y.; Chen, Z.; Han, W. True random coded photon counting Lidar. Opto-Electron. Adv. 2020, 3, 19004401-19004406. [CrossRef]

18. Yu, Y.; Liu, B.; Chen, Z.; Hua, K. Photon Counting LIDAR Based on True Random Coding. Sensors 2020, 20, 3331. [CrossRef] [PubMed]

19. Blaj, G. Dead-time correction for spectroscopic photon counting pixel detectors. J. Synchrotron Radiat. 2019, 26, 1621-1630. [CrossRef] [PubMed]

20. Arvani, F.; Carusone, T.C. Direct Time-of-Flight TCSPC Analytical Modeling Including Dead-Time Effects. In Proceedings of the 2018 IEEE International Symposium on Circuits and Systems (ISCAS), Florence, Italy, 27-30 May 2018.

21. Li, Z.; Lai, J.; Wang, C.; Yan, W.; Li, Z. Influence of dead-time on detection efficiency and range performance of photon-counting laser radar that uses a Geiger-mode avalanche photodiode. Appl. Opt. 2017, 56, 6680-6687. [CrossRef] [PubMed]

22. Hwang, I.P.; Lee, C.H. Mutual Interferences of a True-Random LiDAR with Other LiDAR Signals. IEEE Access 2020, 8, 124123124133. [CrossRef]

23. Hwang, I.P.; Lee, C.H. A rapid LiDAR without Mutual Interferences. In Proceedings of the Optical Fiber Communication Conference, San Diego, CA, USA, 3-7 March 2019.

24. Gatt, P.; Johnson, S.; Nichols, T. Geiger-mode avalanche photodiode ladar receiver performance characteristics and detection statistics. Appl. Opt. 2009, 48, 3261-3276. [CrossRef] [PubMed] 